Schüler als Sanitäter

\title{
Schulsanitätsdienst - Bindeglied zwischen Erster Hilfe und professionellem Rettungsdienst
}

\author{
Uwe Glatz, Eppingen
}

NOTFALLMEDIZIN 2003; 29: 92-97

Kindern schon frühzeitig die Angst vor Notfallsituationen zu nehmen, ist ein wichtiger Ansatz, um die medizinische Laienversorgung zu verbessern. Denn Kinder haben oftmals noch geringe Hemmungen im Umgang mit Verletzten. Sie lernen schnell und können so bei vielen Unfällen effektiv Hilfe leisten. Schulsanitätsdienste leisten hierzu einen wertvollen Beitrag und ermöglichen darüber hinaus eine zeitnahe medizinische Erstversorgung. Dadurch sind Schulsanitätsdienste wichtiges Bindeglied zwischen Erster Hilfe und professionellem Rettungsdienst.

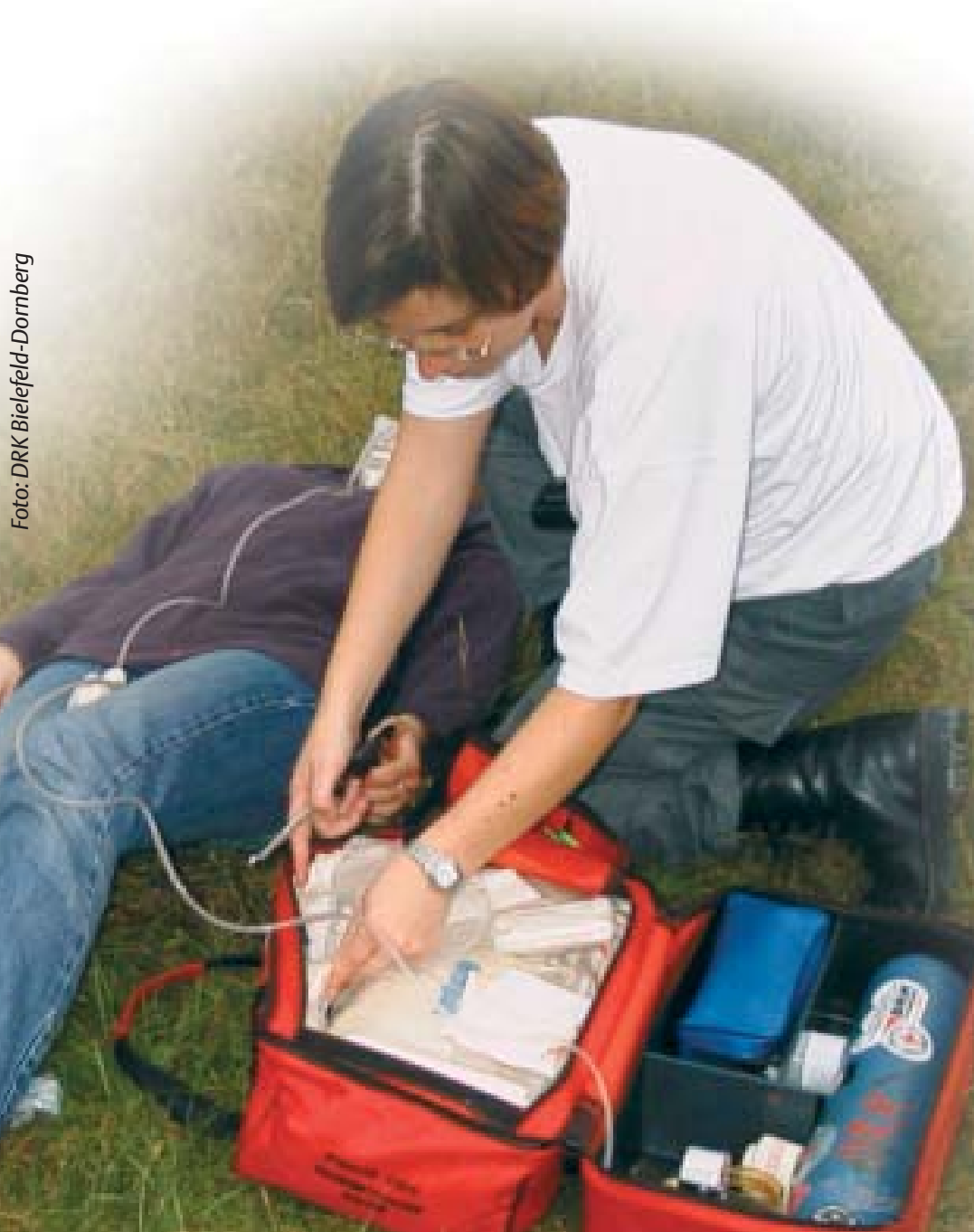

W esentliches Ziel der Notfallversorgung muss es sein, in möglichst kurzer Zeit effektive medizinische Hilfe zu leisten. In Deutschland existiert die Maßgabe, dass innerhalb von 15 Minuten nach der Alarmierung der Rettungsleitstelle ein Rettungswagen, beziehungsweise ein Notarzt am Ort des Geschehens eintreffen sollte. In akut lebensbedrohlichen Situationen kann dies jedoch bereits eine entscheidende Zeitverzögerung bedeuten. Denn leider ist nicht jeder in der Lage, suffizient Erste Hilfe zu leisten und die Zeit bis zum Eintreffen des Rettungsdienstes zu überbrücken. Um bei der Bevölkerung möglichst frühzeitig die Hürden zu überwinden, bei einem Unfall oder sonstigen gesundheitlichen Notfällen aktiv zu werden, erscheint es sinnvoll, bereits Kinder mit dem richtigen Verhalten in einer Notsituation vertraut zu machen.

\section{Im Notfall das Richtige tun}

Zunächst einmal spielt es keine Rolle, wie alt die Kinder sind und welche Schule sie besuchen, um ihnen die Grundlagen der Notfallversorgung zu vermitteln. Bereits im Kindergarten können Kinder lernen, wie kleine Verletzungen behandelt werden können oder wie man schnellstmöglich Hilfe herbeiruft. Diesen Grundgedanken greifen Schulsanitätsdienste auf, die nun mehr und mehr in Schulen aller Art ins Leben gerufen werden. Dabei lernen Kinder und Jugendliche, ihre eigenen Hemmungen zu überwinden und im Notfall das Richtige zu tun. 
- Strukturelle Voraussetzungen

Grundsätzlich hat jede Schule einen ebenerdigen Sanitätsraum bereitzuhalten, um während des Schulbetriebs auftretende gesundheitliche Probleme auffangen zu können. Als Minimalausstattung muss dieser Sanitätsraum über folgende Einrichtungsgegenstände verfügen:

- Warm- und Kaltwasseranschluss

- Liege mit Decke

- Trage mit Decke

- Verbandskasten.

Diese Utensilien erlauben jedoch lediglich, einfachste Verletzungen oder Erkrankungen zu behandeln. Soll der Schulsanitätsdienst wirklich zu einer wesentlichen Verbesserung der Notfallversorgung beitragen, reicht die oben genannte Einrichtung bei weitem nicht aus. So empfehlen die Rettungsdienstorganisationen eine wesentlich umfangreichere Ausstattung (Tab. 1), mit der die Schulsanitäter effektiv Hilfe leisten können.

\section{Was ist ein Schulsanitäts- dienst?}

Ein Schulsanitätsdienst hat das Ziel, im Notfall rasch und kompetent medizinische Hilfe zu gewährleisten. Um an einer Schule einen Schulsanitätsdienst einrichten zu können, muss zunächst ein Lehrer gefunden werden, der sich bereit erklärt, die Organisation, Betreuung und vor allem Verantwortung für das Projekt zu übernehmen. Die Schüler, die am Schulsanitätsdienst teilnehmen, sollten als Grundlage - je nach Alter des Schülers - mindestens einen Junior-Helfer-Kurs bzw. Erste-HilfeKurs absolviert haben, damit sie über Basiswissen der Notfallversorgung verfügen. Darüber hinaus macht es Sinn, wenn ältere Kinder und Jugendliche an einer erweiterten Ersthelfer-Ausbildung teilnehmen.

Wichtige Voraussetzung für den reibungslosen Einsatz im Ernstfall sind regelmäßige Übungen in der Schule, bei denen Verbände, stabile Seitenlage oder auch Mund-zuMund-Beatmung und Herzdruckmassage trainiert werden. Zudem sollten die Mitglieder eines Schulsanitätsdienstes regelmäßig an Auf-

\section{Tab. 1 Empfehlung zur Ausstattung von Schulsanitätsdiensten}

- Tisch und Stühle

- Abschließbarer Schrank

- Abfalleimer

- Kühlschrank mit Gefrierfach

- Rettungsdecken

- Untersuchungshandschuhe in verschiedenen Größen

- Nierenschalen

- Flüssigseife und Handcreme im Spender

- Hände- und Wunddesinfektionsmittel

- Einmalhandtücher

- Würfelzucker, Traubenzucker

- Kühl-Packs

- Stethoskop

- Blutdruckmanschette

- Verbandsscheren

- Diagnostikleuchten

- Thermometer

- Pinzetten

- Taschenbeatmungsmasken

- Zahnrettungsbox

- Guedel-Tuben in verschiedenen Größen

- Augenklappen

- Heftpflaster

- Wundschnellverbände

- Kompressenvorrat (10x10 cm)

- Verbandspäckchen in verschiedenen Größen

- Mundspatel

- Wärmflasche

- Sicherheitsnadeln

- Warnwesten

- Notfalltasche/ -koffer

- Artikel der Monatshygiene

frischungskursen teilnehmen. Solche Seminare bieten die örtlichen Abteilungen der Rettungsdienstorganisationen an, die nicht selten auch einen Mitarbeiter als externen Berater oder Betreuer für den Schulsanitätsdienst zur Verfügung stellen.

\section{Synergieeffekte nutzen}

Eine Kooperation mit diesen Verbänden kann außerdem zu Synergieeffekten führen, da möglicherweise interessierte Jugendliche für eine aktive Mitarbeit in den Jugendabteilungen der Organisationen gewonnen werden können. Auf der anderen Seite kann ein Schulsanitäts-

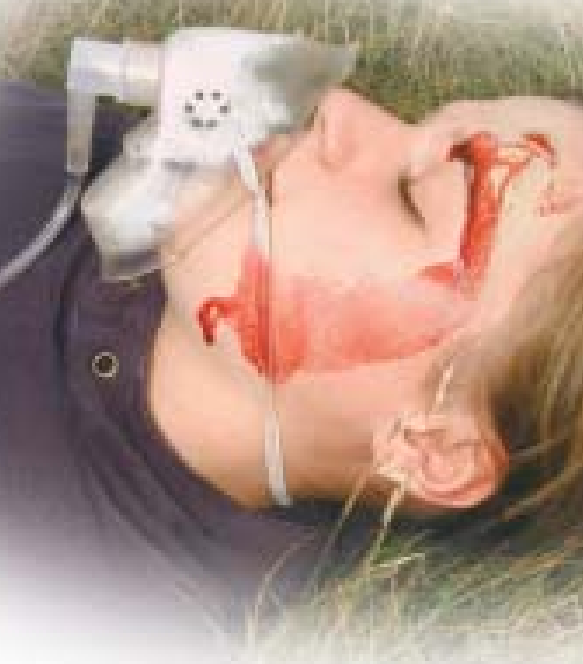

dienst auch davon profitieren, wenn sich Schüler darin engagieren, die durch ihre Mitgliedschaft in Rettungsdienstorganisationen bereits über Erfahrungen in der Notfallversorgung verfügen.

\section{Verletzungsspektrum in der Schule}

Häufige Erkrankungen in Schulen und Kindergärten sind in aller Regel kleine Verletzungen wie Platzund Schürfwunden sowie Verstauchungen. Unfallschwerpunkte sind in diesen Fällen vor allem Werk- und Sportunterricht. Hinzu kommen internistische Krankheitsbilder wie Gastroenteritis, ein entgleister Diabetes mellitus Typ I oder ein akuter Asthmaanfall. Aber auch andere Probleme wie Ohrenschmerzen, Augenverletzungen oder Dysmenorrhoe fallen in das Spektrum der Erkrankungen, die in einer Schule auftreten können.

Sicherlich sind nicht alle Verletzungen oder Erkrankungen, die sich in einer Schule oder Kindergarten ereignen, so schwer wiegend, dass der Rettungsdienst oder Notarzt verständigt werden muss. Aber auch bei schweren Unfällen lernen die Kinder und Jugendlichen, wie man in solchen Situationen adäquat reagiert.

\section{Verschiedene \\ Alarmierungsmöglichkeiten}

Tritt ein gesundheitlicher Notfall in der Schule auf, müssen die Schulsanitäter umgehend alarmiert werden. Dies kann über verschiedene Alarmierungssysteme erfolgen, die folgende Eigenschaften erfüllen müssen und je nach örtlichen Gege- 
benheiten Vor- und Nachteile mit sich bringen:

- Einfaches und schnelles System: Die Sanitäter müssen ohne großen Aufwand rasch erreichbar sein;

- Kostengünstiges System: Das System sollte in Anschaffung und Unterhaltung den Anforderungen der jeweiligen Schule entsprechen;

- Sicherheit: Das System muss garantieren, dass die Schulsanitäter auch tatsächlich erreicht werden.

Eine Möglichkeit besteht darin, dass die Schulsanitäter durch so genannte Melder benachrichtigt werden. Bei dieser Variante ist es Voraussetzung, dass in allen Klassenzimmern und Fachräumen der Dienstplan des Schulsanitätsdienstes aushängt. Daraus ist ersichtlich, welche Schüler Bereitschaftsdienst haben und wo sie in welcher Schulstunde und welcher Pause zu finden sind. Werden in einer Klasse Sanitäter benötigt, laufen Mitschüler („Melder“) des verletzten Kindes los und holen die Sanitäter aus den entsprechenden Räumen. Probleme können sich bei kurzfristigem Wechsel der Klassenräume und Erkrankung eines Schulsanitäters ergeben.

Weitere Alternativen bieten unterschiedliche technische Einrichtungen wie Funkrufsysteme („Pieper“, z.B. Scall), schnurlose Telefonanlagen oder Funkgeräte. Welche dieser Möglichkeiten zum Einsatz

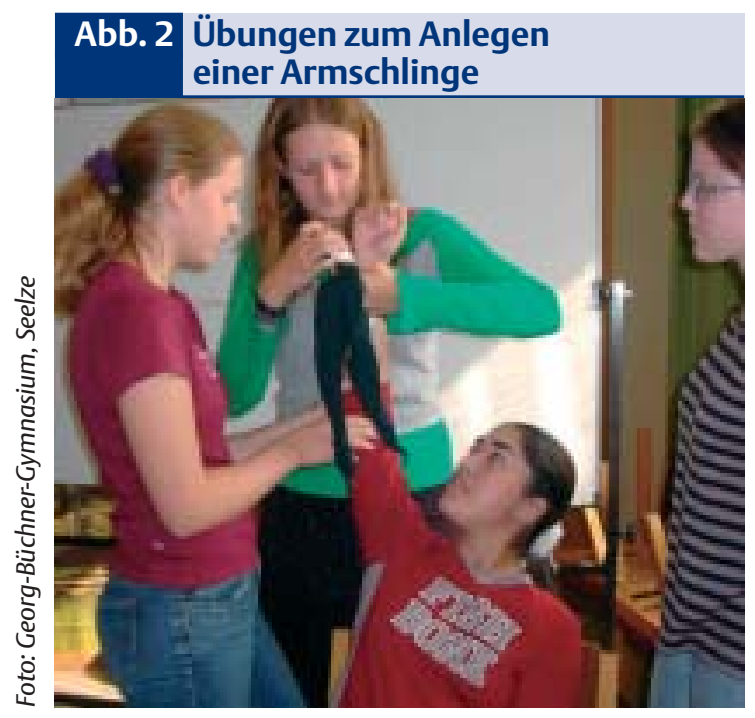

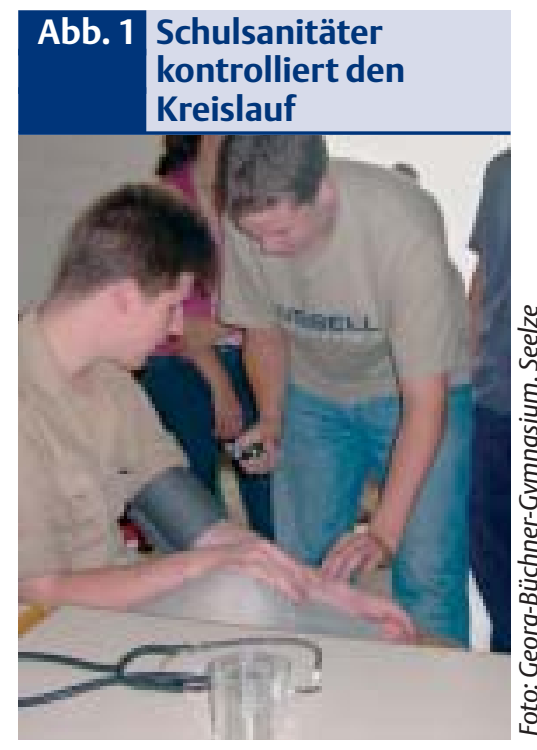

kommt, hängt von diversen Faktoren wie beispielsweise Größe der Schule, Kosten der Anlage oder Erreichbarkeit der Empfänger ab. Eine kostengünstige Variante stellt eine fest installierte Durchsageanlage dar, die jedoch den Nachteil mit sich bringt, dass im Einsatzfall der Unterricht in allen Klassen gestört wird.

\section{Altersabhängige Aufgaben}

In Abhängigkeit vom Alter erhalten die Kinder bestimmte Einsatzgebiete zugewiesen. So können Jugendliche zum Beispiel - nach entsprechender Einweisung - auch ohne direkte Aufsicht kleine Wunden im Sinne der Ersten Hilfe versorgen oder bei einem verunfallten Kameraden die Kreislaufüberwachung übernehmen (Abb. 1). Kleinere Kinder können Pflaster kleben, Tränen abwischen und Hilfe herbeirufen. Überhaupt ist die psychologische Betreuung ein wesentlicher Bestandteil der medizinischen Versorgung von Kindern. Denn sehr häufig werden Kinder durch die Angst vor der außergewöhnlichen Situation mehr beeinträchtigt als durch die Verletzung an sich. Beruhigende Worte können daher oft Wunder bewirken.

\section{Vorstellung beim D-Arzt}

Steht die Entscheidung an, ob der betroffene Schüler den Unterricht fortsetzen kann oder ob dieser vom Unterricht befreit werden muss, oder liegt eine lebensgefährli- che Situation vor, ist auf alle Fälle der zuständige Lehrer zu informieren. Dieser entscheidet über das weitere Vorgehen, nimmt mit den Eltern und - falls erforderlich - mit dem medizinischen Fachpersonal außerhalb der Schule (Haus- oder Kinderarzt bzw. Rettungsdienst) Kontakt auf und organisiert die weitere Versorgung des jungen Patienten.

Dabei ist zu beachten, dass die Schüler während des Unterrichts sowie auf dem Schulweg über den Schulträger unfallversichert sind. Daher darf eine eventuell notwendige ärztliche Erstbehandlung nur durch einen so genannten Durchgangsarzt (D-Arzt) erfolgen, der gegenüber der jeweiligen Unfallversicherung behandlungsberechtigt ist. Dies sind in erster Linie niedergelassene Chirurgen oder Orthopäden, chirurgische Notfallambulanzen in den Krankenhäusern sowie Augen- und HNO-Ärzte oder Kliniken bei entsprechenden Verletzungen. Ausgenommen von dieser Regelung ist die erforderliche Erstbehandlung durch den Notarzt.

Wird der erkrankte Schüler nach Hause entlassen, sollte dieser niemals ohne Begleitung den Heimweg antreten. Können die Eltern ihn nicht von der Schule abholen, sollte immer eine weitere Begleitperson für den Heimweg vom Unterricht freigestellt werden.

\section{Wer darf was?}

Die Aufgaben und Tätigkeiten der Schulsanitäter beschränken sich keineswegs nur auf Basismaßnahmen der Ersten Hilfe („Basic life support“) wie zum Beispiel Schocklagerung, stabile Seitenlage, Wundverbände (Abb. 2) oder Mund-zuMund-Beatmung und Herzdruckmassage. Nach entsprechenden Schulungen dürfen Schulsanitäter auch erweiterte Maßnahmen (,advanced cardial life support“) ergreifen.

So können unter anderem bei einem Herzkreislaufstillstand auch Automatisierte Externe Defibrillatoren (AED) zum Einsatz kommen. Dabei müssen jedoch Empfehlungen der Bundesärztekammer eingehalten werden, wonach nur Personen 
einen AED anwenden sollen, die eine entsprechende Einweisung erhalten haben (www.bundesaerztekammer.de/30/Richtlinien/Empfidx/NotfallD.html).

Keine selbstständige Verordnung von Medikamenten

Schulsanitäter dürfen Kreislaufkontrollen mit Blutdruck- und Pulsmessungen durchführen oder den Blutzucker bestimmen. Außerdem können sie einem Mitschüler Medikamente verabreichen, die dieser wegen seiner Grunderkrankung bereits von einem Arzt verordnet bekam und ständig bei sich trägt. Typische Beispiele sind die Applikation eines Dosieraerosols bei einem Asthmaanfall oder die Gabe von Glukose bei einem Typ-I-Diabetiker im Unterzucker.

Nicht erlaubt ist jedoch das selbstständige Verordnen von Medikamenten. Dies ist und bleibt Aufgabe des behandelnden Arztes. Darüber hinaus findet auch die für professionelle Rettungsassistenten gültige „Notkompetenz“ bei den Schülern keine Anwendung.

Nachbesprechung ist wichtig

Jeder Einsatz des Schulsanitätsdienstes muss sorgfältig protokolliert werden. Die verwendeten Formulare (Abb. 3 ) ähneln denjenigen des professionellen Rettungsdienstes. So wird gewährleistet, dass auch im Nachhinein der Einsatz noch nachvollzogen werden kann.

Jedem Einsatz sollte auch eine dem Ereignis angemessene - Nachbesprechung folgen. Auf diese Weise können die beteiligten Schüler Fehler aufdecken, Probleme besprechen und möglicherweise auftretenden Traumatisierungen durch den Einsatz vorbeugen.

\section{Wichtiges Bindeglied}

Grundsätzlich ersetzt der Schulsanitätsdienst nicht den Einsatz des öffentlichen Rettungsdienstes. Insbesondere wenn der Verdacht besteht, dass sich der verletzte Schüler ernstere oder gar lebensbedrohliche Verletzungen zugezogen hat, darf nicht gezögert werden, nach Rücksprache mit dem verantwortlichen Lehrer umgehend den Rettungs-

\section{Abb. 3 Einsatzprotokoll für den Schulsanitätsdienst}

Einsatzprotokoll

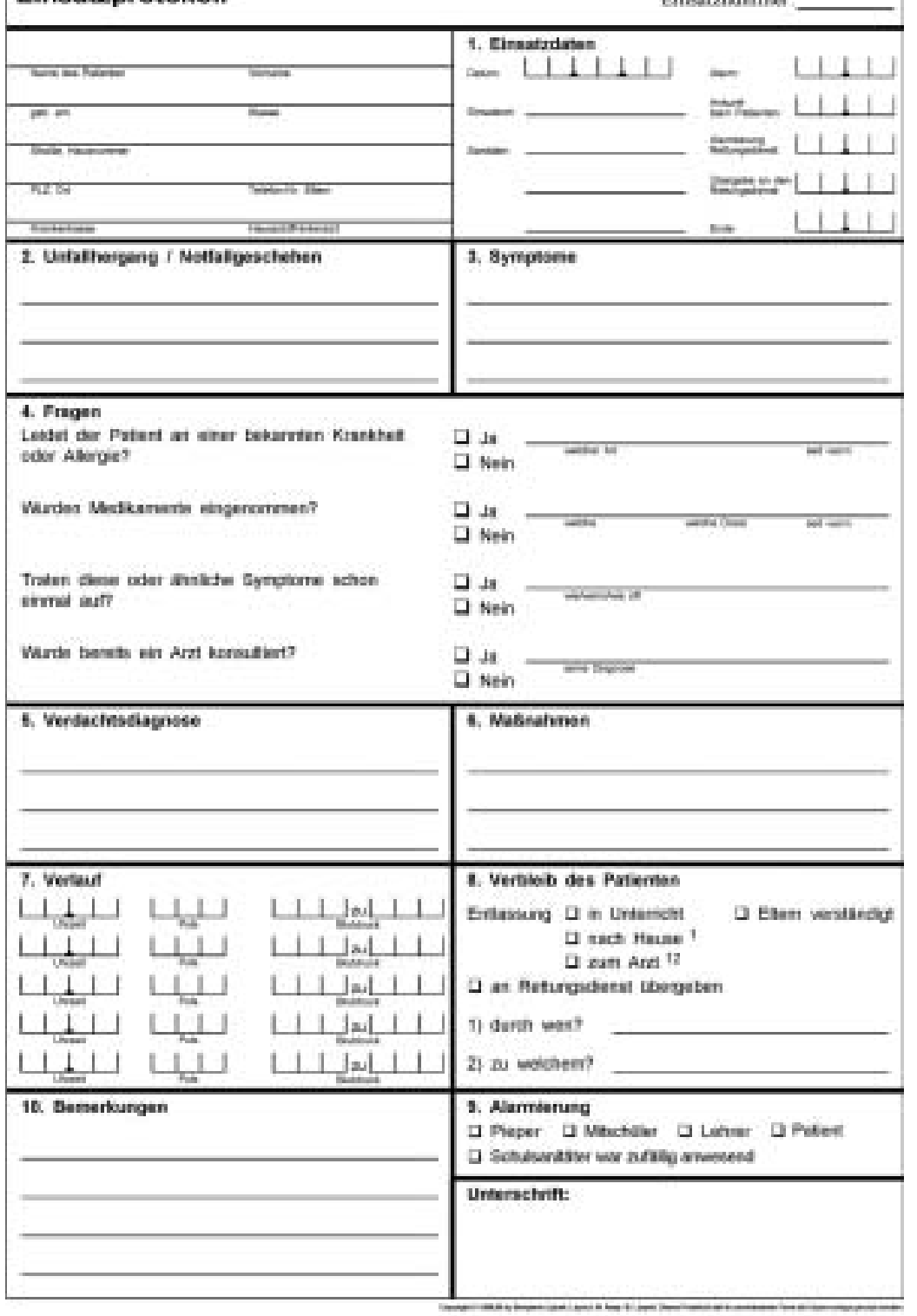

dienst zu alarmieren. Während professionelle Hilfe unterwegs ist, können die Schulsanitäter jedoch bereits mit den erforderlichen Maßnahmen beginnen. So kann wertvolle Zeit eingespart werden, die unter Umständen lebensrettend sein kann. Schulsanitätsdienste können auf diese Weise ein wichtiges Bindeglied zwischen Erster Hilfe und professionellem Rettungsdienst sein.

\section{Summary}

A major approach to improving provision of medical care by laypersons is to relieve children at an early age from their fear of an emergency situation.
For children often have fewer reservations about approaching an injured person. They learn quickly, and can give effective aid in numerous accident situations. In this connection, school-based medical services are in a position to make a valuable contribution, and can also provide first aid quickly. This makes these services important links between official first aid posts and the professional medical rescue services.

\section{Anschrift des Verfassers}

Uwe Glatz

Weisenbergstr. 34

75031 Eppingen

Uwe.Glatz@epost.de 